\title{
An efficient non-occluded face area detection method under unconstrained environment of smartphone database
}

\begin{abstract}
Occlusion is one of the most challenging problems for recognizing faces captured in unconstrained conditions. This paper focuses on direct detect the useful part in the face which used for features extraction process. To solve occlusion problem and detect un occluded face parts, a Non-Occluded Face Area Detection (NOFAD) method based on geometric features is proposed. The performance of the proposed method is evaluated on the SFV and MOBIO databases, and the experimental results show the outperformance of the proposed method in extracting the non-occluded facial area, realising an accuracy rate of $99.4 \%$ and $99.1 \%$, respectively.
\end{abstract}

Keyword: Face detection; Face recognition; Occlusion; Geometric features; Smartphone database 